\title{
Formation of cold clumps and filaments around superbubbles
}

\author{
E. Ntormousi ${ }^{1}$, P. Hennebelle ${ }^{1}$, J. Dawson ${ }^{2}$ and F. Del Sordo ${ }^{3}$
}

${ }^{1}$ Laboratoire AIM, Paris-Saclay, CEA/IRFU/SAp - CNRS - Universit Paris Diderot, 91191, Gif-sur-Yvette Cedex, France email: eva.ntormousi@cea.fr

${ }^{2}$ Department of Physics and Astronomy and MQ Research, Centre in Astronomy, Astrophysics and Astrophotonics, Macquarie University, NSW 2109, Australia

${ }^{3}$ Department of Geology \& Geophysics, Yale University, PO Box 208109, New Haven CT 06520-8109, USA

\begin{abstract}
The majority of young massive stars are found in close binary systems. Recently, dedicated observing campaigns have provided strong constraints on the binary fraction as well as the distribution of the parameters that characterize the binary systems: the masses of both components, the orbital period and eccentricities. Most strikingly these findings imply that the majority of massive stars experience strong interaction (roche lobe overflow, a common envelope phase and or a merger) with a binary companion before their final explosion. I will discuss recent results from detailed binary star models and population synthesis models.
\end{abstract}

